\title{
Using video to support veterinary nursing students preparing for OSCEs.
}

\author{
Emily J. Hall MA VetMB AFHEA MRSB MRCVS
}

Emily qualified as a veterinary surgeon in 2007, and has worked in small animal first opinion practice ever since. Emily now teaches veterinary nursing students on the Foundation degree at Nottingham Trent University, and continues to work in practice at weekends.

\section{Alison Simpson RVN TAQA AFHEA}

Alison qualified as a veterinary nurse in 1994, working in small animal practice before moving into veterinary nursing education. Alison has also worked for the National Blood Service, and examined for the RCVS. She joined Nottingham Trent University in 2008 as Veterinary Nursing Centre Manager, responsible for overseeing practical skills teaching and assessment.

\section{Introduction}

Objective structured clinical examinations (OSCEs) inspire fear and loathing in both student veterinary nurses and clinical coaches alike. With different courses using different OSCE assessment criteria and different styles of assessment, students can find preparing for their OSCEs stressful and confusing if they receive different advice from difference sources. A further source of anxiety may be a perceived element of unfamiliarity with the equipment and resources likely to be present in the examination; if a student uses one type of centrifuge in practice, but then faces a different model in their OSCE this can trigger panic, and shaking hands may struggle to fill a capillary tube, especially with the added pressure of an examiner armed with a clipboard standing in attendance!

\section{Background}

At Nottingham Trent University, we use a scenario based OSCE assessment for all three years' practical assessments. Students face a number of practical tasks, all linked to a fictional patient, with a time limit to complete the whole scenario. This style of assessment allows a little more flexibility for students, they don't have to be able to complete all practical skills in under 6 minutes. The downside to this style of OSCE, is that an earlier upset can spell disaster if the student dwells on their mistakes and loses concentration. To try and support students through this challenging assessment, we have explored ways of making the OSCE assessment more transparent, and as suggested by Heywood (2017), de-mystifying the assessment process. 
Our current course structure places the final OSCE assessment at the end of a year-long placement period. The hope is that having spent a year in practice performing the day one skills, students arrive at the final hurdle confident and ready to demonstrate their practical abilities. The reality is that students worry about picking up "bad habits", and panic that they won't remember how to use the equipment that differs to their placement practice - different models of centrifuge and anaesthetic machines can be an additional, unnecessary challenge in an OSCE setting. As confidence and self-belief are required to pass this assessment (Heywood, 2017), we have spent the last few years developing practical skills videos to try and support students in preparing for this assessment.

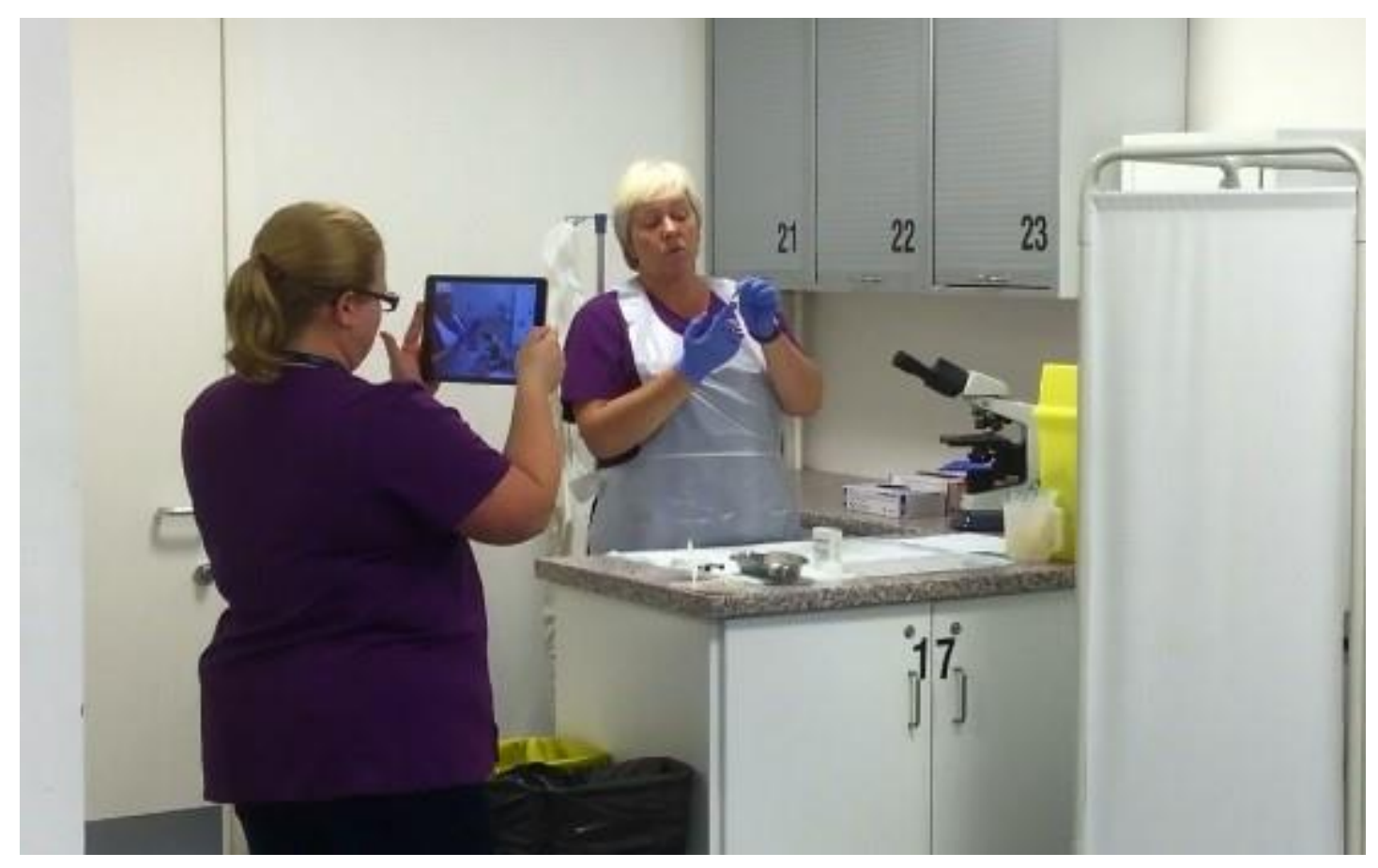

Figure 1: Recording an OSCE station using an assessment set up.

Creating video resources can be costly, time consuming and unrewarding if they are never used. For this reason, we have refined our practical skills demonstration videos into two styles: a formal, OSCE station demonstration using an assessment setting (see figure 1), and an informal, in-class tutorial often recorded by our students within a practical session. The formal OSCE demonstrations are most popular with the final year students, and feedback suggests they help improve familiarity with the task, the equipment, the OSCE station set up and provide students with an idea of how quickly they should be aiming to complete the task. In contrast to some medical demonstration recommendations (George and Doto, 2001), our students prefer narrated videos over silent demonstrations. We therefore talk through the tasks, highlighting key points and clarifying where needed. Recording these demonstrations 
requires time and planning. Whilst it may take less than 10 minutes to record the demonstration, the room will need to be set up and the station prepared as per assessment standards. The best time to record these demonstrations is before, or after an assessment day, or during examination moderation or standardisation processes. With practice, and familiarity with the workflow of recording to sharing a video, we can record 10 tasks, and have them visible on the virtual learning environment (VLE) in two working days.

The in-class demonstrations, are basically "lecture capture" for practical classes. Students are asked to play the role of cameraperson, and the demonstration provided at the beginning of a session can be recorded within the session, meaning there is no additional time required to set up the task and the recording is completed within a timetabled session requiring only one tutor. These demonstrations have the advantage of recording clarification questions from both the tutor, and the students, providing additional information that may not be presented in the OSCE style demonstration. Allowing students access to these demonstration recordings during revision and placement periods, provides constant access to refresher tutorials if needed.

\section{Video recording using an iPad - a workflow model}

\section{Step one.}

Choose your location. If you are recording an in-class demonstration, your location may be limited by your timetable restrictions. If you are planning to record a number of OSCE station recordings, then it may be ideal to consider recording your videos on a day when you have a number of OSCEs already set up. We often video tasks after a mock examination session, as the stations are all set out already. In an ideal world, you would pick an area with a plain, preferably pale background (the medical privacy screens we use in OSCEs are ideal for this), and minimal distractions.

\section{Step two.}

Choose your task. This sounds obvious, but decide what you want to video, and make sure the demonstrator is familiar with the steps! Recording post OSCE assessing is ideal, as having spent the day watching students complete the task the methodology is likely to be ingrained on your memory. This is also an ideal time to record common mistakes. Students really value seeing mistakes demonstrated and identified, so they can recognise any bad practice they might have inadvertently developed (Hall and Stewart, 2017). It is also 
important that the cameraperson knows the task, so they can stop you if required to re-start, and so they know when to zoom in to key manual tasks.

\section{Step three.}

Set up your recording device. Check your iPad has enough storage for your video. There is nothing more frustrating that getting 5 minutes through a demonstration, and the iPad flashing up "insufficient storage". It is also worth checking your camera settings, to ensure your video will be good quality. Whilst you could spend thousands of pounds on high quality camera and sound equipment, in our experience, the quality from an iPad or tablet is perfectly acceptable to the students, and means we have easy access to recording devices at no extra cost.

\section{Step four.}

Record your demonstration. Feedback from our students suggested some tips for improving the usefulness of OSCE recordings (see figure 2).

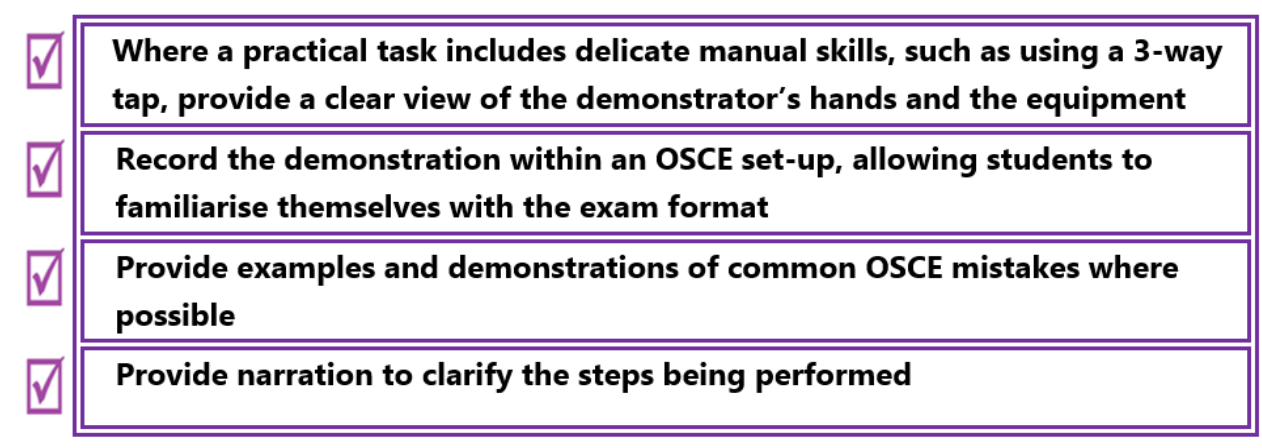

Figure 2: Student recommendations for developing OSCE video resources (Hall and Stewart, 2017).

If possible, try to record in one take. Trimming videos down is easy, combining two or three videos together requires video editing software and time, so it is quicker and simpler to just stop the demonstration and start again.

\section{Step five.}

Upload your video. You may choose to edit your video before this point but honestly, it is easier to trim, stabilise and correct lighting once the video is on YouTube (or which ever video sharing media you choose to use). If you have recorded using a tablet or iPad, and your device is connected to a reasonable internet connection, it is usually possible - and simplest - 
to upload the video straight to YouTube from here. You will need a Google account to upload videos to YouTube, but this is free, and you can use your work e-mail address to sign up. We use unlisted as the privacy setting, as this prevents the videos appearing in searches, but means anyone with the link can view the recording. This is a personal decision you need to make about how far you would want the recording to be disseminated.

\section{Step six.}

Edit and stabilise. If you have used a hand-held camera, your video will be shaky. YouTube can correct this for you, alongside providing a trimming tool (See figure 3), so you can edit out sections if needed and you only need an up to date internet browser to perform this editing.

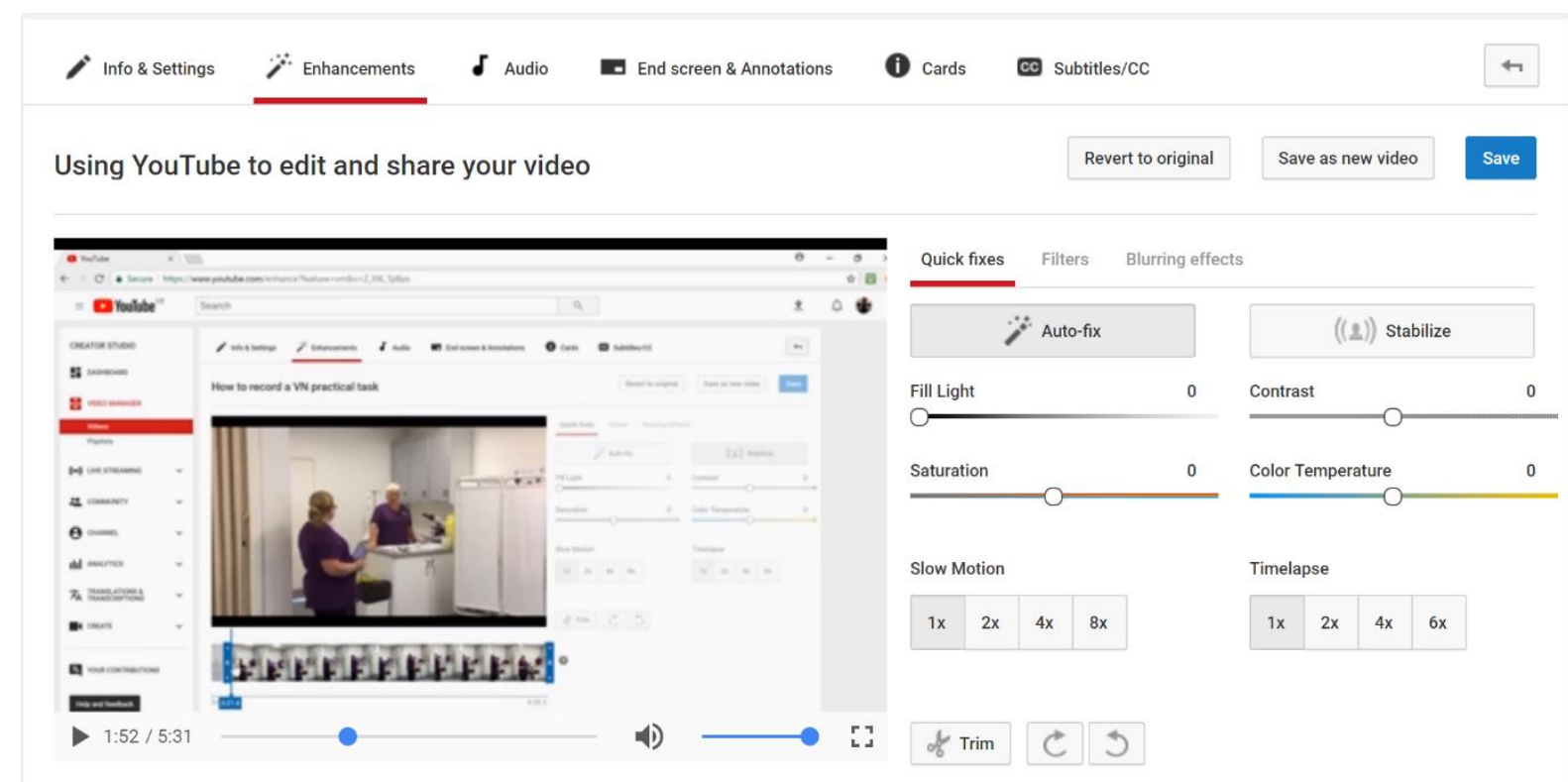

Figure 3: The YouTube editing tools useful for removing camera shake and trimming videos.

\section{Step seven.}

Share your work. If you are creating resources for students, chances are you will have a virtual learning environment to upload, or embed videos. Embedding videos has the advantage of not using up your VLE's storage capacity. Embedding the videos also has the advantage that students can select the quality of the video they view, so if they have poor internet speed at home, they can choose to view a lower quality video that will load faster.

For video demonstrations of these steps, please see the supplementary material available with the on-line version of this article. 


\section{Conclusion}

OSCEs are stressful. Removing the mystery behind OSCE grading is a key way to try and reduce student assessment anxiety (Byrne and Smyth, 2008), so providing video demonstrations of the tasks performed by the assessors, using the equipment that will be present in the assessment can help to improve familiarity with the practical tasks (Massey et al., 2017). Where students are on placement a great distance from the training centre, it can be both financially, and logistically difficult for them to attend multiple revision sessions, meaning these students may have less opportunity to familiarise themselves with the equipment they are likely to face in their assessment. On-line video resources ensure all students have access to centre specific revision materials, no matter where they are based, or when they are free to revise. Recording the videos is also a great way to check tutor standardisation, and can be used for OSCE examiner standardisation training too.

\section{References}

Byrne, E. and Smyth, S. (2008). Lecturers' experiences and perspectives of using an objective structured clinical examination. Nurse Education in Practice, 8(4), pp. 283-9.

George, J.H. and Doto, F.X. (2001). A simple five-step method for teaching clinical skills. Family Medicine, 33, 577-588.

Heywood, A. (2017). OSCEs hints, tips and tricks for students and clinical coaches. Veterinary Nursing Journal, 32(2), 54-56.

Hall, E.J. and Stewart, A. (2017, October). What do final year veterinary nursing students want from a virtual learning environment during their final year work placement? Poster presented at the British Veterinary Nursing Association Congress, Tamworth, UK.

Massey, D., Byrne, J., Higgins, N., Weeks, B., Shuker, M., Coyne, E., Mitchell, M. and Johnston, A. (2017). Enhancing OSCE preparedness with video exemplars in undergraduate nursing students. A mixed method study. Nurse Education Today, 54, 56-61. 